\title{
Bichromatic Synthetic Schlieren applied to surface wave measurements
}

\author{
Jostein Kolaas $^{\mathrm{a}}$, Bjørn Hervold Riise ${ }^{\mathrm{a}, \mathrm{b}}$, Kristian Sveen ${ }^{\mathrm{a}, \mathrm{c}}$, Atle Jensen ${ }^{\mathrm{a}, *}$ \\ ${ }^{a}$ Department of Mathematics, University of Oslo, Oslo, Norway \\ ${ }^{b} D N V$ GL, Oslo, Norway \\ ${ }^{c}$ Insitute for Energy Technology, Kjeller, Norway
}

\begin{abstract}
The present paper provides an introduction to Bichromatic Synthetic Schlieren (BiCSS) for surface measurements, a novel extension of the Free Surface Synthetic Schlieren (FS-SS) by Moisy et al. (2009). The new technique is based on the fact that light diffraction through a medium varies with wavelength. Therefore, one may apply light at two different wavelengths to measure the change in density gradient in a medium. This paper explores the use of the difference between blue visual and near-infrared (NIR) light, but the choice of wavelengths will typically depend on the application. Calibration was performed using stationary targets of plexiglass and the results show that the new BiCSS technique improves accuracy for large surface gradients, compared to the traditional FS-SS technique. In order to test the applicability of the technique in the laboratory, two sets of experiments were performed. Firstly, an experiment using phase-locked regular waves was conducted for comparing BiCSS with FS-SS, analyze the properties and give an estimation of the error. Secondly, to investigate the applicability for more complex surface patterns, a study on a vertical surface-piercing cylinder exposed to a focused wave was conducted, obtaining the complex surface characteristics. The new technique clearly reveals nonlinear wave diffraction, in addition to cross waves and parasitic capillary waves.
\end{abstract}

Keywords: Bichromatic Synthetic Schlieren, Free Surface Synthetic Schlieren, experiments, cylinder, wave-structure interaction, nonlinear wave diffraction

\section{Introduction}

Computational Fluid Dynamics (CFD) has, in the last 3 decades, become a standardized tool in both academic research and industrial engineering. However, in many cases, the available laboratory data for validation are limited or simply not available. One such example is the measurement of a 3-dimensional wave field. In marine hydrodynamics, for example, one of the key questions concern the measurement of surface characteristics and complex wave phenomena due to the presence of a structure.

One example is the offshore wind industry, where the monopile is by far the most commonly used support structure for wind turbines. Furthermore, the offshore oil industry has extensively applied offshore platform designs based on either one, three or four legged designs.

For a cylinder in waves, the characteristics of the disturbed surface have typically been studied either by images from high-speed video (see e.g. Chaplin et al., 1997; Rainey, 2007; Kristiansen and Faltinsen, 2017), or by the use of wave gauges, obtaining point measurements of the elevation (see e.g. Sheikh and Swan, 2005; Chen et al., 2014; Swan and Sheikh, 2015). Considerable work is ongoing, investigating the problem numerically, with the development of CFD, see e.g. Paulsen et al. (2014a,b); Chen et al. (2014). Both for improved understanding of the problem, and providing comparison data for computational modeling efforts detailed measurements of a disturbed surface is useful.

The present work concerns the conceptual aspects of a novel technique, which provides a detailed description, on a previously unattainable level, of a measured free surface. The method is based on non-intrusive optical pattern matching techniques, such as Synthetic Schlieren (Sutherland et al., 1999; Dalziel et al., 2000) and Background Oriented Schlieren (Meier, 2002). The general procedure for these types of optical methods is by the use of a camera to capture images of a fixed pattern through a volume

\footnotetext{
*Corresponding author

Email addresses: josteiko@gmail.com (Jostein Kolaas), bjorn.riise@gmail.com (Bjørn Hervold Riise), Kristian.Sveen@ife.no (Kristian Sveen), atlej@math.uio.no (Atle Jensen) 
of fluid, and measure the displacement of the pattern between the refracted and a reference image. Historically, these techniques were used to obtain the density field by relating the pattern displacement to the variation in refractive index through the volume.

The concept was later used by Moisy et al. (2009), who developed Free Surface Synthetic Schlieren (FS-SS), for free surface measurements, making use of the step-like change in the refractive index at the free surface. The displacement between the refracted image visualized trough a deformed interface and a reference image of a flat surface, obtains the surface gradient, which is used to calculate the surface elevation.

We further extend the methodology by introducing Bichromatic Synthetic Schlieren (BiCSS). The new technique uses the difference between two simultaneous images taken using two different wavelengths of light to obtain the change in refractive index. This is in contrast to the original SS/BOS/SS-FS technique which is based on measuring the difference between a reference image taken at a time, $t_{0}$, and a subsequent image. In the present investigation we have chosen to maximise the difference between the two wavelengths by using blue and near infrared (NIR) light, but we note that for even larger variations in refractive index, for example blue and red (visible) would possibly suffice.

The new developments provides an improvement in resolving small scale structures of the surface, when large scale structures are present, typically shorter waves traveling on longer waves and other small disturbances due to the presence of a structure.

Previously NIR light has been used for surface measurements without the use of a pattern, by taking advantages of absorption of light, by combining red and NIR illumination. Jähne (1990) obtained the wave slope by refraction, using two alternating light source with linear intensity change through a diffusion box. Later Jähne et al. (2005) extended the technique, obtaining the surface elevation using absorption and the wave slope by refraction. More recently Aureli et al. (2014) further developed the concept for use in non-horizontal bottoms. These methods rely on light sources with a small difference in the lights wavelengths, as the refraction angle is assumed to be the same for both lights. By using high speed cameras, Kiefhaber et al. (2014) was able to create a more robust slope refraction method for surface measurements based on intensity ratios only.

Other alternative methods for measuring spatial surface elevation, can be done by using reflection and polarization of light, see e.g. Zappa et al. (2008). Furthermore, using reconstruction of stereo images, nice results have been obtained from a moving vessel by Benetazzo et al. (2016).

The present work is a follow up on a previous work by Kolaas et al. (2016). The present work extends the previous work with new in-depth analyses, including a study of a vertical surface-piercing cylinder exposed to a focused wave. The measurements of the disturbed surface, due to the presence of a structure, highlight the excellence of the technique in resolving the small scale surface characteristics, due to the wave-structure interaction. The measurements of the entire instantaneous disturbed surface around the cylinder compared to the corresponding undisturbed surface, reveals nonlinear wave scattering, including both Type I and Type II wave scattering (Sheikh and Swan, 2005; Swan and Sheikh, 2015). In addition, the method is able to capture other aspects like cross waves due to resonance of the wave flume, and parasitic capillary waves observed in the front of the wave. The new technique is considered to be a good contribution for future investigations, both to improve our understanding of the complex surface characteristics due to wave-structure interaction, and for providing comparisons data for computational modeling efforts.

The paper is organized as follows: section 2 gives a short overview of FS-SS and describes the new BiCSS technique, section 3 presents the experimental setup, section 4 shows a calibration study using a solid plexiglass surface, and section 5 discusses the experimental results.

\section{Methodology}

\subsection{Free Surface Synthetic Schlieren}

One of the challenges when using Synthetic Schlieren for free surface measurements, is to relate the displacement of the pattern to the surface gradient. In Free Surface Synthetic Schlieren (FS-SS), as characterized by Moisy et al. (2009), the pattern displacement $\delta r$ is found with regards to a reference image from a flat surface. In Moisy et al. (2009) derivation of the relation between $\delta r$ and the instantaneous free surface gradient $\nabla h$ three approximations where considered: i) paraxial approximation, requiring that the distance from the camera to the pattern is much larger than the field of view; ii) weak slope approximation, requiring that the slope of the measured surface is small; and iii) weak amplitude 
approximation, requiring that the wave amplitude is much smaller than the apparent distance from still water level to the pattern. Then it was shown that $\nabla h$ can be expressed by a linear equation

$$
\nabla h=-\frac{\delta \mathbf{r}_{\lambda}}{h_{\lambda}^{*}}, \quad \text { with } \quad \frac{1}{h_{\lambda}^{*}}=\frac{1}{\alpha_{\lambda} h_{p, \lambda}}-\frac{1}{H},
$$

where $\alpha_{\lambda}=1-\frac{n_{a, \lambda}}{n_{w, \lambda}}$, a function of the refractive index of air $n_{a, \lambda}$ and water $n_{w, \lambda}$, and $H$ is the distance from the camera to the pattern. The refractive index is depending on the wave length of the light, which is denoted by the subscript $\lambda$. The apparent distance from the still water level to the pattern $h_{p, \lambda}$ is given by

$$
h_{p, \lambda}=h_{w}+\frac{n_{w, \lambda}}{n_{g, \lambda}} h_{g}+\frac{n_{w, \lambda}}{n_{a, \lambda}} h_{a}
$$

where $h_{w}$ is the water depth, which is adjusted for the tank bottom thickness $h_{g}$, with refractive index $n_{g, \lambda}$, and the air gap between the wave flume and the pattern, $h_{a}$, as shown in figure 1 .

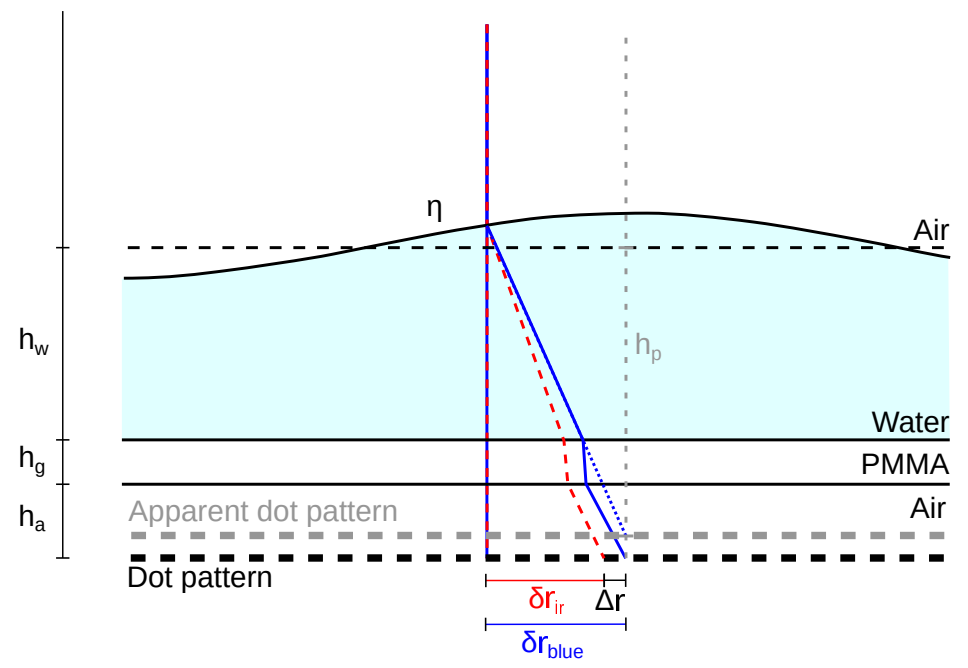

Figure 1: BiCSS principle. The diffraction angles are highly exaggerated for better illustration of the concept. During the linearization of the reconstruction problem the bottom of the flume and air gap between the flume and the pattern are simplified to an equivalent case with an apparent dot pattern at water depth $h_{p}$.

In the present study the refractive indices was estimated using Sellmeier dispersion formula (Sellmeier, 1871) given by

$$
n^{2}-1=\sum_{i=1}^{4} \frac{A_{i} \lambda^{2}}{\lambda^{2}-\lambda_{i}^{2}},
$$

where $n$ is the refractive index and $\lambda$ is the wavelength of the light. The coefficients $A_{i}$ and $\lambda_{i}$ are determined by fittings to experimental data from Daimon and Masumura (2007), Ciddor (1996) and Sultanova et al. (2009) for water, air and PMMA, respectively. Selected values for the refractive indices

\begin{tabular}{|c|c|c|c|}
\hline & $\begin{array}{c}\text { Water } \\
n_{w, \lambda}\end{array}$ & $\begin{array}{c}\text { Air } \\
n_{a, \lambda}\end{array}$ & $\begin{array}{c}\text { PMMA } \\
n_{g, \lambda}\end{array}$ \\
\hline Blue $\quad(\lambda=470 \mathrm{~nm})$ & 1.3384 & 1.0002798 & 1.4979 \\
\hline Red $\quad(\lambda=660 \mathrm{~nm})$ & 1.3314 & 1.0002762 & 1.4880 \\
\hline$(\lambda=880 \mathrm{~nm})$ & 1.3273 & 1.0002746 & 1.4835 \\
\hline
\end{tabular}
are shown in table 1.

Table 1: Refractive indices for selected wavelengths and materials 


\subsection{Bichromatic Synthetic Schlieren}

In Bichromatic Synthetic Schlieren (BiCSS), using both visual blue and near infrared (NIR) light, the pattern displacement $\Delta \mathbf{r}$ is found, using the difference in refraction between the two wavelengths, by

$$
\Delta \mathbf{r}=\delta \mathbf{r}_{\lambda_{\text {blue }}}-\delta \mathbf{r}_{\lambda_{\text {nir }}}
$$

where $\delta \mathbf{r}_{\lambda_{\text {blue }}}$ and $\delta \mathbf{r}_{\lambda_{\text {nir }}}$ are the individual diffraction angles, see figure 1. Inserted (4) into (1) for each wavelength of the lights, $\lambda$, and rearranging the terms we get a similar linear equation for the gradient of the instantaneous free surface

$$
\nabla h=-\frac{\Delta \mathbf{r}}{h_{\lambda_{\text {blue }} \lambda_{\text {nir }}}^{*}}
$$

with $h_{\lambda_{\text {blue }} \lambda_{\text {nir }}}^{*}=h_{\lambda_{\text {blue }}}^{*}-h_{\lambda_{\text {nir }}}^{*}$.

Using the difference between two wavelengths puts a stricter requirement on the paraxial approximation, which may result in a displacement when the surface is at rest, $\Delta \mathbf{r}_{\mathbf{0}}$. This requires a correction in the equation for the surface gradient (5),

$$
\nabla h=-\left[\frac{\Delta \mathbf{r}}{h_{\lambda_{b l u e}^{*} \lambda_{n i r}}^{*}}-\frac{\Delta \mathbf{r}_{\mathbf{0}}}{h_{\lambda_{b l u e} \lambda_{n i r}}^{*}}\right] .
$$

In theory, when a telecentric lens is used, the paraxial approximation is fulfilled per definition. Given that all light rays are perpendicular to the flat surfaces and if there is no chromatic aberration in the lens, there will be no need of a pair of reference images as the correction term will vanish as $\left|\Delta \mathbf{r}_{0}\right| \rightarrow 0$ as $H \rightarrow \infty$. In practice the lens usually has some aberrations and some correction may be necessary. The coordinate system would also be a fixed property of the CCD size and lens, giving the possibility of a calibration-free measurement system.

\subsection{Reconstruction of the surface elevation}

Bichromatic Synthetic Schlieren obtains the surface gradient $\nabla \eta=\nabla h$. To reconstruct the surface elevation $\eta(\mathbf{x}, t)$, where $\mathbf{x}$ is the horisontal coordinate and $t$ the time, the gradient field may be integrated by

$$
\eta(\mathbf{x}, t)=\int_{C} \nabla \eta(\mathbf{s}, t) \cdot d \mathbf{s}+\eta\left(\mathbf{x}_{0}, t\right),
$$

where $\mathrm{C}$ is any path from $\mathbf{x}_{0}$ to $\mathbf{x}$, and the elevation in a single point $\eta\left(\mathbf{x}_{0}, t\right)$ is left as an undetermined function of time.

To determine $\eta\left(\mathbf{x}_{0}, t\right)$ more information or additional constraints are needed. Making a narrowbandwidth approximation, the surface elevation may be expressed by (Toffoli et al., 2010)

$$
\eta(\mathbf{x}, t)=\eta_{0}+A e^{\mathrm{i} \chi}+A_{2} e^{\mathrm{i} 2 \chi}+A_{3} e^{\mathrm{i} 3 \chi}+\ldots+c . c,
$$

where $\eta_{0}$ is the mean water level, $A$ the slowly modulated amplitude, $\chi=\mathbf{k} \cdot \mathbf{x}-\omega_{c} t$ the phase function, $\mathbf{k}$ the wave number, $\omega_{c}$ the characteristic wave frequency, $A_{m}$ (for $m=2,3$. .) higher order components, and $c . c$ is the complex conjugate. It can be shown that to leading order the temporal derivative can be expressed using spatial derivatives

$$
\frac{\partial \eta}{\partial t}=\frac{\partial x}{\partial t} \frac{\partial \eta}{\partial x}+\frac{\partial y}{\partial t} \frac{\partial \eta}{\partial y}=-\left(c_{x} \frac{\partial \eta}{\partial x}+c_{y} \frac{\partial \eta}{\partial y}\right),
$$

where $c_{x}=\omega_{c} / k_{x}$ and $c_{y}=\omega_{c} / k_{y}$ is the components of the phase velocity. For waves with wave propagating in one direction only, i.e. $c_{y}=0$, the surface elevation can be evaluated as

$$
\eta\left(\mathbf{x}_{0}, t\right)=\int \frac{\partial \eta}{\partial t}\left(\mathbf{x}_{0}, t\right) d t=-c_{x} \int \frac{\partial \eta}{\partial x}\left(\mathbf{x}_{0}, t\right) d t
$$

Inserting this into (7) yields

$$
\eta(\mathbf{x}, t)=\int_{C} \nabla \eta(\mathbf{s}, t) \cdot d \mathbf{s}-c_{x} \int \frac{\partial \eta}{\partial x}\left(\mathbf{x}_{0}, t\right) d t+\eta_{0},
$$


which reduces the unknowns to two constants: mean water level $\eta_{0}$, which is easily measurable, and the characteristic wave propagation velocity $c_{x}$, which may be slowly varying in time and can be estimated using cross-correlation methods.

When the surface elevation is reconstructed by integration of the gradient an uncertainty is introduced related to the obtained constant $\eta_{0}$ and $c_{x}$. This uncertainty is not relevant for the obtained surface gradients. In present work the description of the waves, given the above assumptions, is assumed sufficient for determine the unknown constants. However, the BiCSS technique is not restricted to narrow banded wave spectra, nor unidirectional waves.

The constant $\eta_{0}$ and $c_{x}$ is determined assuming unidirectional wave propagation. This requires that there are small reflections of the waves in the wave flume. The reflection index $R I$ is found using the method outlined by Goda and Suzuki (1977). See appendix A for more details. For the regular waves used in the phased-locked error estimation, the wave damper works reasonable well with $R I \approx 0.1$. For the focusing waves the damping is of less importance since the measurements are taken before any reflection reaches the measurement section.

For present work, the gradient field has been integrated using "Inverse (integrated) gradient" (D'Errico, 2006). Before the integration, the measured gradients were filtered by a forth order Butterworth high pass filter with a cut-off frequency of $\omega_{c}=3.14 \mathrm{rad} / \mathrm{s}$ in time to prevent mean water level drifting.

\section{Experimental setup}

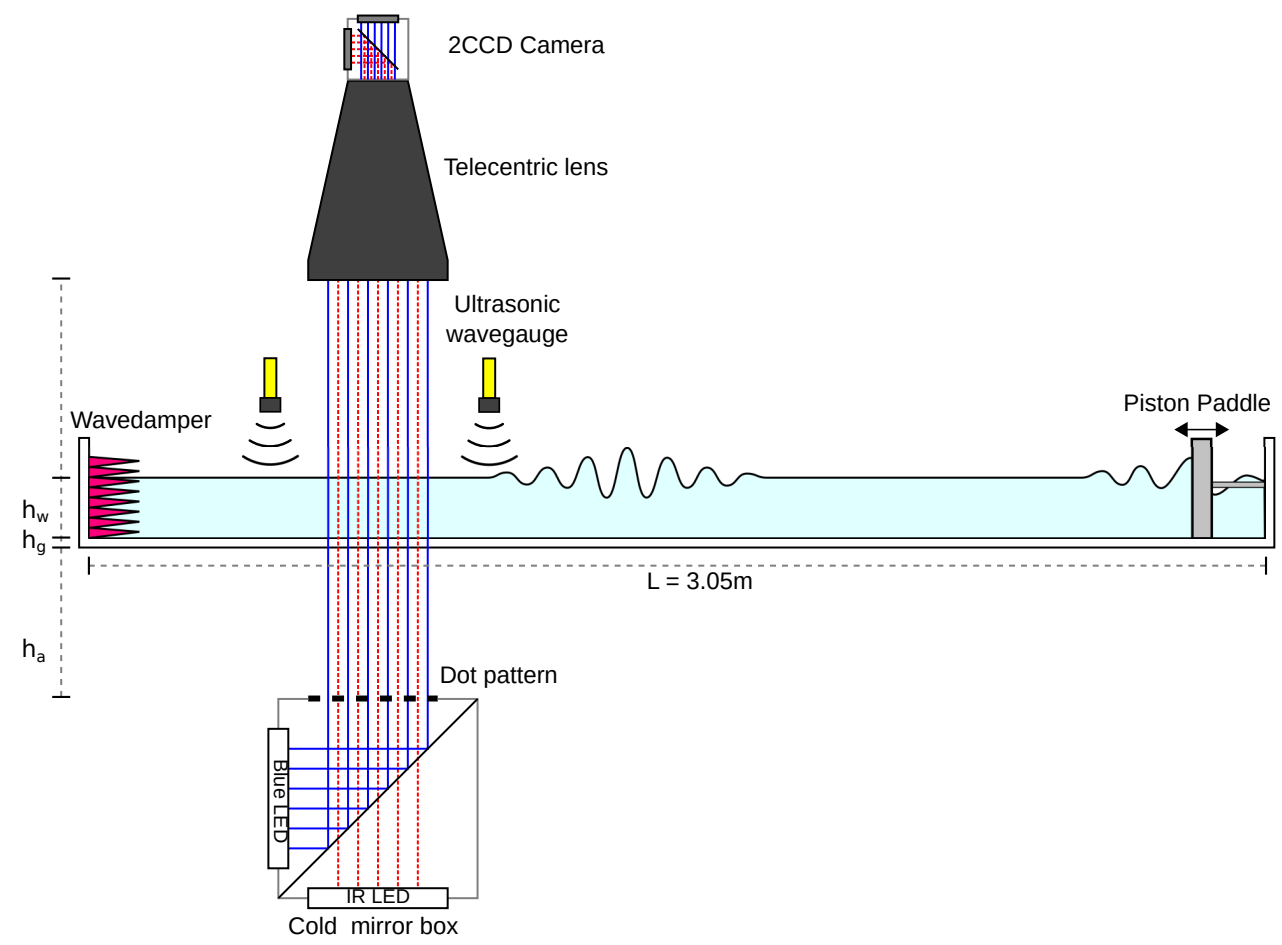

Figure 2: Overview of the experimental setup showing wave flume, wavemaker, wavedamper and a test section consisting of a bichromatic LED based light source box, a 2CCD camera with telecentric lens and ultrasonic wave gauges.

In order to test the technique in the laboratory, experiments were carried out in the Hydrodynamic Laboratory at the University of Oslo, in a small scale wave flume, $305 \mathrm{~cm}$ long and $10 \mathrm{~cm}$ wide, see figure 2. The wave flume, made of transparent PMMA plastic with wall thickness $h_{g}=6 \mathrm{~mm}$, was filled to a water depth of either $h_{w}=50 \mathrm{~mm}$ or $h_{w}=62.5 \mathrm{~mm}$. In one end of the flume a piston-type wavemaker was used to generate regular and focusing waves. The opposite end contained a wavedamper, consisting of many narrow spikes with a length of $150 \mathrm{~mm}$, see appendix A.

At a location of $190 \mathrm{~cm}$ from the wavemaker, a transparent dot pattern was placed below the wave flume. The pattern consisted of dots with a diameter of $225-335 \mu \mathrm{m}$, which were randomly spaced with a concentration of $1.84 \mathrm{dots} / \mathrm{mm}^{2}$. The flume was placed on a traversing table, enabling adjustment of the apparent pattern distance $h_{p, \lambda}$ by adjusting the air gap between the flume and the fixed pattern 


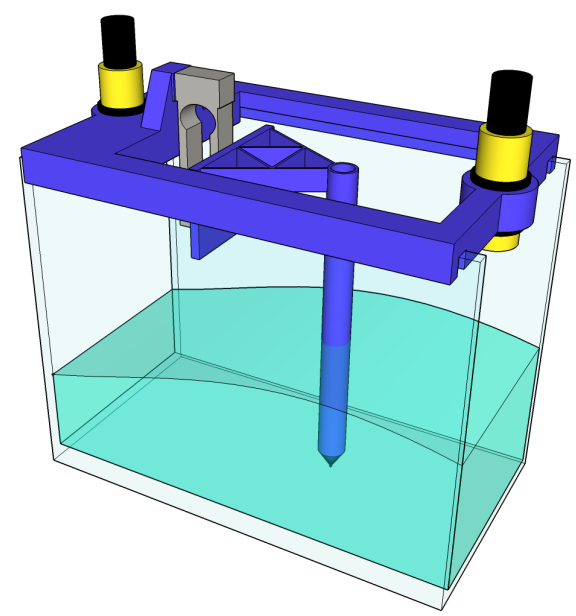

Figure 3: Close up view of the test section showing the cylinder mounted to frame through a load cell (Gray) for force measurement and the Ultrasonic wave gauges (Yellow/Black)

$h_{a}$. The pattern was illuminated using two Advanced illumination BL0404 4"x4" Surface Mount LED Back Lights, with peak wavelength 470nm, blue visual light, and 880nm, near-infrared light (NIR). The two individual wavelengths of the lights were then combined using a cold mirror, which reflects the blue visual light, while the NIR passes through. The LED lights were pulsed at $30 \mathrm{~Hz}$ with a pulse width of 750 us.

To record the deformation of the pattern, a Visual/NIR Camera (jAi AD-130 GE) equipped with a telecentric lens (OPTO Engineering TC12144) was focused on the pattern. The camera separates the visual spectrum from near-infrared into 2 charge-coupled devices (CCDs) using a hot prism. The visual CCD has a Bayer-filter, so the camera records the image in four channels, red, green, blue and near-infrared, in which only the blue and infrared channel were used in the experiment. The lens used in this experiment does have some chromatic aberration and is not completely telecentric and thus needs correction for the displacement when the surface is at rest. It is noted that the use of the telecentric lens was primarily dictated by practicalities (compact setup) rather than high scientific standards. In practice a standard camera lens would probably have sufficed.

In a second set of experiments, a vertical surface-piercing cylinder with a diameter $D=12.5 \mathrm{~mm}$ was placed in line with the center axis of the pattern and camera. The wave-exciting force on the cylinder was measured using a strain gauge load cell, PW4MC3/500-G1, with a RM4220 amplifier, both from Hottinger Baldwin Messtechnik GmbH. The load cell mounts the cylinder to a frame, see figure 3 . The frame is also containing two Banner engineering corp U-Gage S18U ultrasonic wave gauges, with a range of 30-300mm for comparing the measured surface elevation.

The in-house particle image velocimetry code, HydrolabPIV (Kolaas, 2016), using multipass with window distortion was used to find the displacement of the pattern. The BiCSS measurement was processed using two passes of 32x32 pixel and two passes using distortion with 32x32 pixel subwindows. To handle the much larger displacements, FS-SS used a cascade of increasingly smaller subwindows, starting with two passes of $256 \times 256$ pixels reduced to $32 \times 32$ pixels via two iterations on $128 \times 128$ and $64 x 64$ pixels respectively, all using $75 \%$ overlapping interrogation windows. Distorted passes were also tested for FS-SS, but was not found to improve the results.

There is a slight difference in shape of the displacement distribution between the two methods, especially the steepest part are underrepresented for FS-SS and there is a trend toward zero gradients, see figure 4. Overall this results in a more smoothed surface. This is likely an effect of the high pixel displacement, which is not optimal for the pattern matching algorithm, that is the pattern matching is poor in areas close to reaching the Invertibility Condition. We note that it is not possible to reduce the displacements by moving the pattern closer as there is no more space. Some of the differences may also be due to nonlinear behaviour that has been neglected in the linearization of the reconstruction problem. There is also a small oscillation in the displacement distribution for FS-SS which indicates some peak-locking, though it is not significant. 

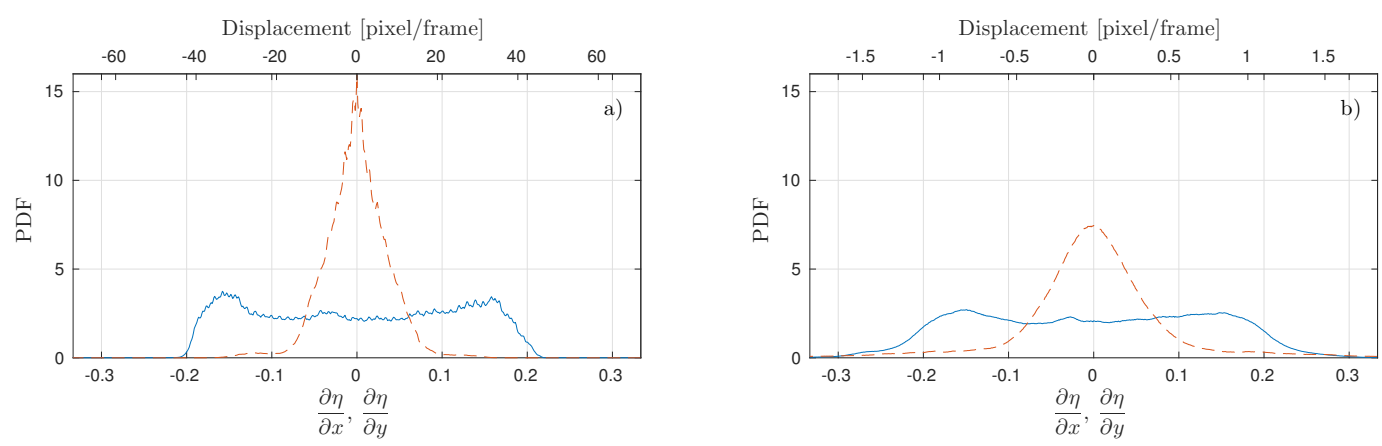

Figure 4: Probability density function (PDF) of the local steepness, $\frac{\partial \eta}{\partial x}(-)$ and $\frac{\partial \eta}{\partial y}(--)$, for regular waves using a) FS-SS and b) BiCSS.

\section{Calibration}

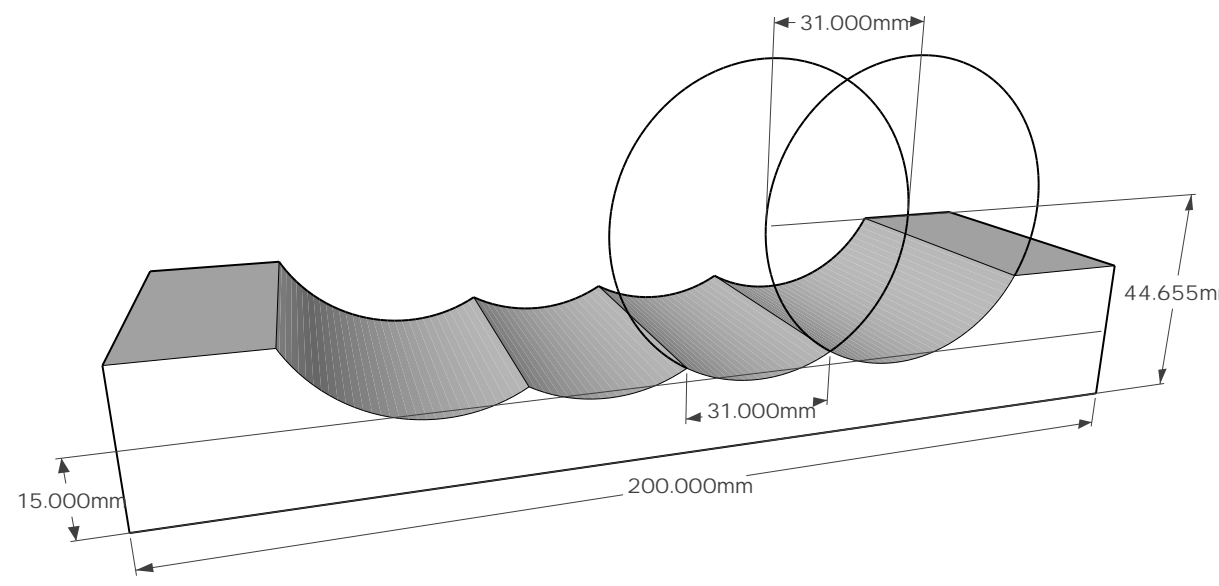

Figure 5: The plexiglass calibration target.

In order to test the BiCSS technique, two stationary targets were manufactured from plexiglass. The first was a planar surface of $15 \mathrm{~mm}$ thickness and the second shaped as shown in figure 5 with a synthetic wave-field with mean thickness of $15 \mathrm{~mm}$. The two targets were imaged and used to compare the measured surface gradient of the synthetic wave field using both SS-FS and BiCSS. The surface gradient results are shown in Figure 6. We observe that the novel BiCSS technique improves the measurement accuracy near the crest regions. At this point, with high curvature, the rays will cross. However, BiCSS is able to resolve this feature with higher accuracy compared to FS-SS. This shows that BiCSS is able to overcome the Invertibility Condition (Moisy et al., 2009)

$$
h_{p, \lambda}<\frac{1}{\alpha_{\lambda} k a_{0}}, \quad \text { where } \eta=a_{0} \cos (k x) .
$$

Here FS-SS is limited by the maximum pattern distance for a sinusoidal wave to avoid crossing of light rays. This can be seen as an even more strict requirement for a weak slope. Overcoming the Invertibility Condition allows measurements where the depth of the water is larger than the maximum pattern distance $h_{w}>h_{p, c}$ when using regular free-surface synthetic schlieren. Note that an alternative solution to this problem has been proposed by Gomit et al. (2013), involving the use of an extra camera and a light sheet.

Figure 7 shows the reconstructed surface profiles based on the measured gradient fields in Figure 6 . The figures shows four measurements of the center-line using different dot patterns, compared with the given surface in red. Unsurprisingly, the reconstructed surface profiles confirm the observation that the BiCSS technique improves the measurement accuracy near the high curvature regions at wave crests, but it is worth noting that the FS-SS technique shows superior accuracy in the regions of low gradients at the 

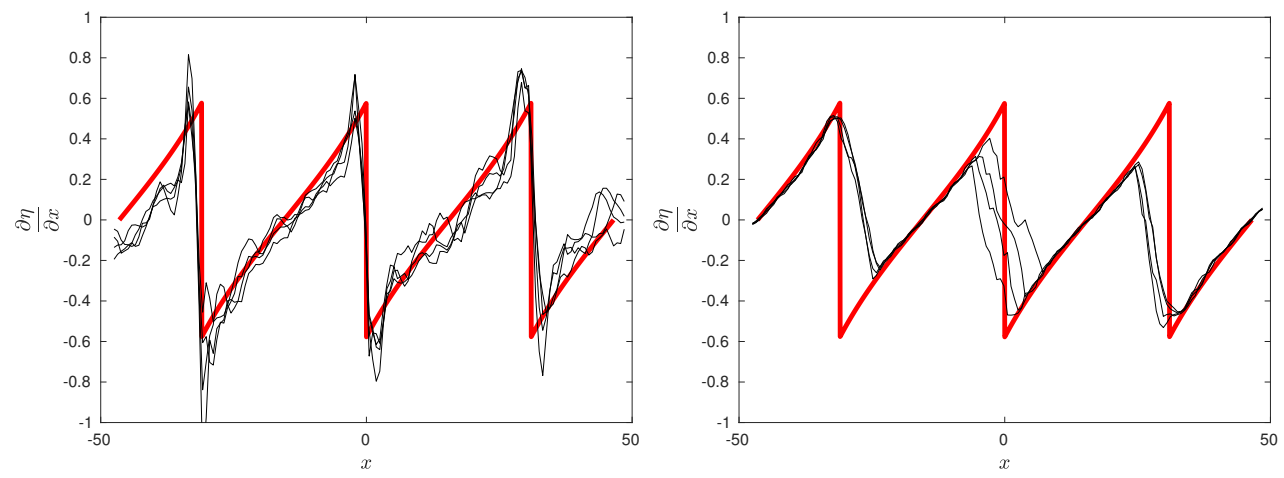

Figure 6: Surface gradient measurements using BiCSS and FS-SS.
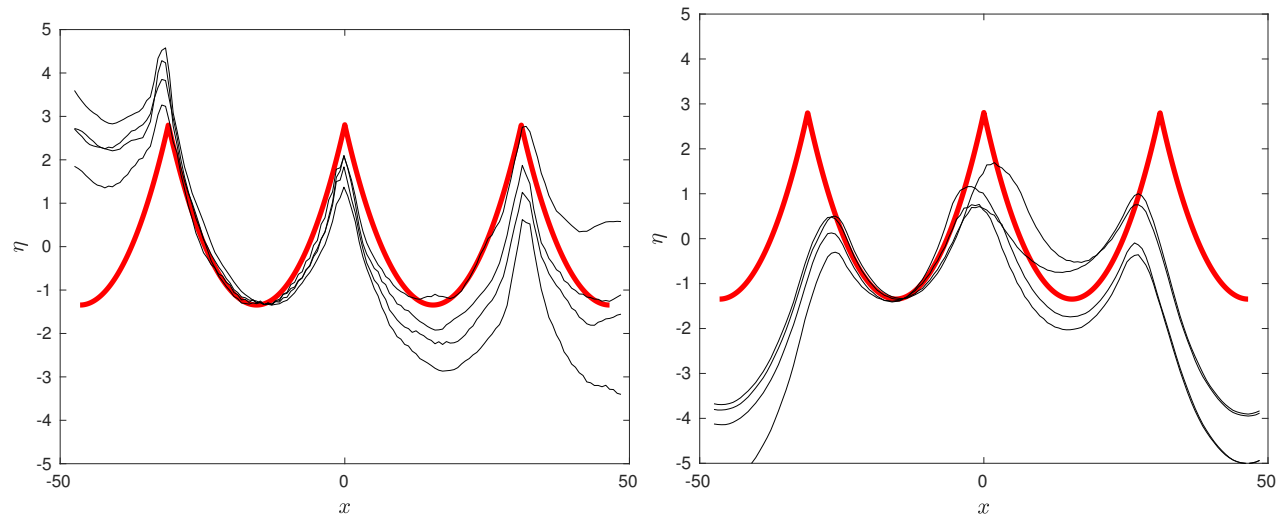

Figure 7: Surface reconstruction using BiCSS (left) and FS-SS (right).

wave troughs (for $-25.3<x<-6$ in Figure 7). Herein, the root mean squared error is $0.13 \pm 0.06 \mathrm{~mm}$ for FS-SS, which is larger than the error stated by Paquier et al. (2015). It should be noted that the present test case is considerably more demanding with respect to the linearization approximations. Alternatively, the errors could also be influenced by the limitation of setting the reference point and angle of the plexiglass block that is used to make the calibration target. This will result in a misalignment of the milling cutter compared to the reference coordinate system. For comparison the error is $0.29 \pm 0.13 \mathrm{~mm}$ for BiCSS.

\section{Experimental results}

\subsection{Phase-locking on periodic waves}

A first set of experiments were conducted to be able to estimate the error when using the method for determining the integration constant described in section 2.3. This method is needed when extending BiCSS (and FS-SS) to measure waves with longer wavelengths than the field of view. A test case generating regular waves with frequency $\omega_{0}=18.85 \mathrm{rad} / \mathrm{s}$ and water depth $h_{w}=50 \mathrm{~mm}$ was conducted. Furthermore, to put the new method into a broader context and show improvements and disadvantages over other methods, the results were compared to the surface geometry obtained by FS-SS. This comparison will include inaccuracies both in the integration constant and the surface elevation.

Since the phase velocity $c$ is constant for a periodic regular wave, it is possible to estimate a phaselocked average in the reference frame following the phase-velocity

$$
\bar{\eta}\left(x^{\prime}, y\right)=\frac{\sum_{i=1}^{N} w\left(\left|x^{\prime}+c t_{i}\right| ; \varepsilon\right) \eta\left(x^{\prime}+c t_{i}, y, t_{i}\right)}{\sum_{i=1}^{N} w\left(\left|x^{\prime}+c t_{i}\right| ; \varepsilon\right)}
$$



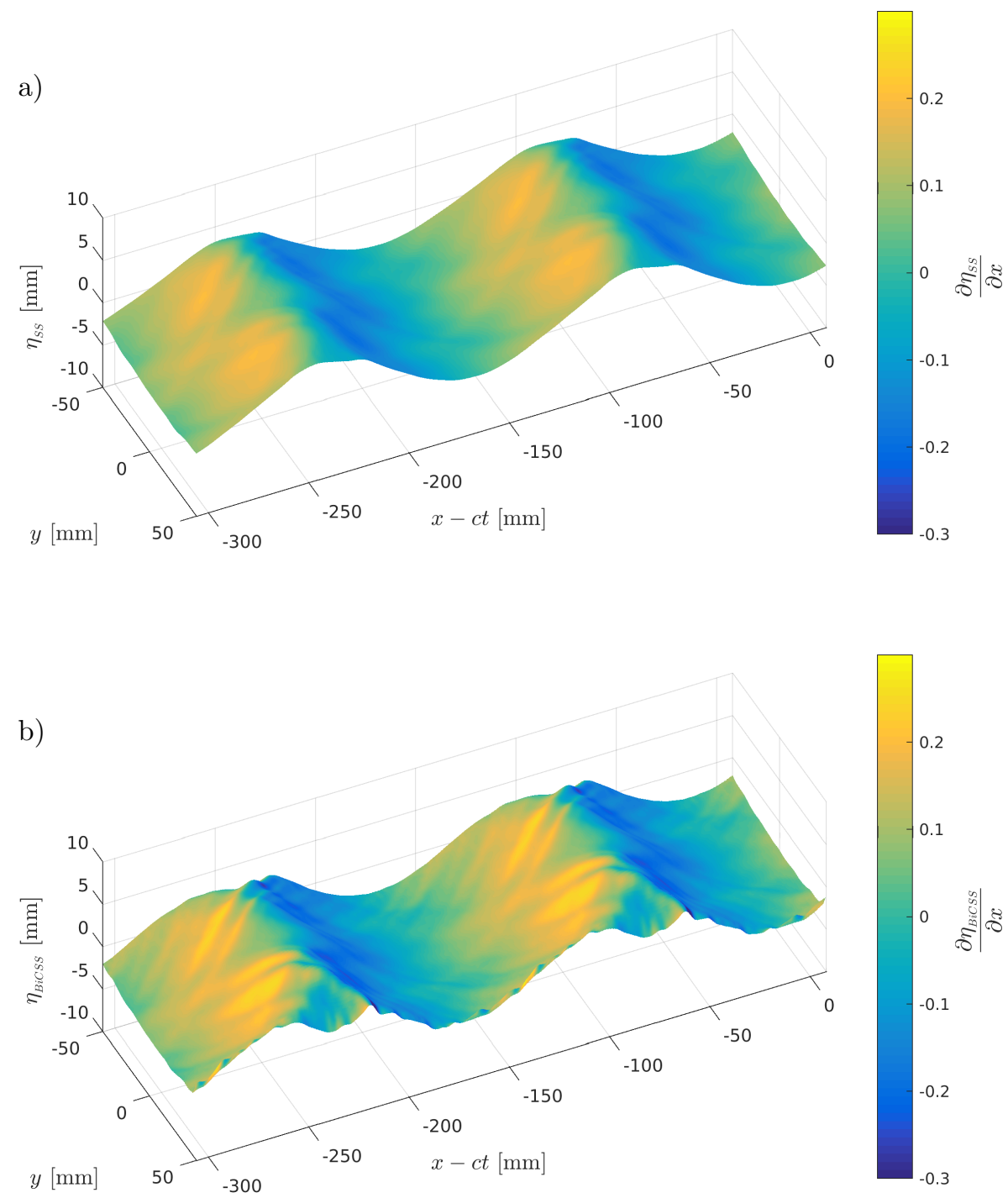

Figure 8: Phase-locked averaged sinusoidal waves for a) Free Surface Synthetic Schlieren (FS-SS) and b) Bichromatic Synthetic Schlieren (BiCSS). The color bar gives the surface gradient in the propagation direction, enchanting the smaller structures on the surface.

where $x^{\prime}=x-c t$ and weight function $w(r ; \varepsilon)=\max (|1-\varepsilon r|, 0)^{4}(4 \varepsilon r-1)$. The parameter $\varepsilon$ is set such that the weight is maximum at the center and drops off to zeros at the boundaries of the field of view. It should be noted that any weight function that goes nicely towards zero can be used.

From the estimate it can be seen that BiCSS keeps the details more intact, see figure 8. For example, the cross-waves which are forming X-shaped structures on the surface (further discussed in section 5.2) appears more refined when compared to FS-SS, where the smaller scales are smoothed out.

To improve measurements of a certain wave length in FS-SS, it is possible to adjust the pattern distance. However, this distance will not be optimal for the entire range, furthermore the nearest distance is physically limited by the wave flume. The position of the pattern is less critical for BiCSS, making it a reasonable choice for experiments containing a wide range of scales.

To further investigate the accuracy of the method it is useful to evaluate the phase-locked standard 

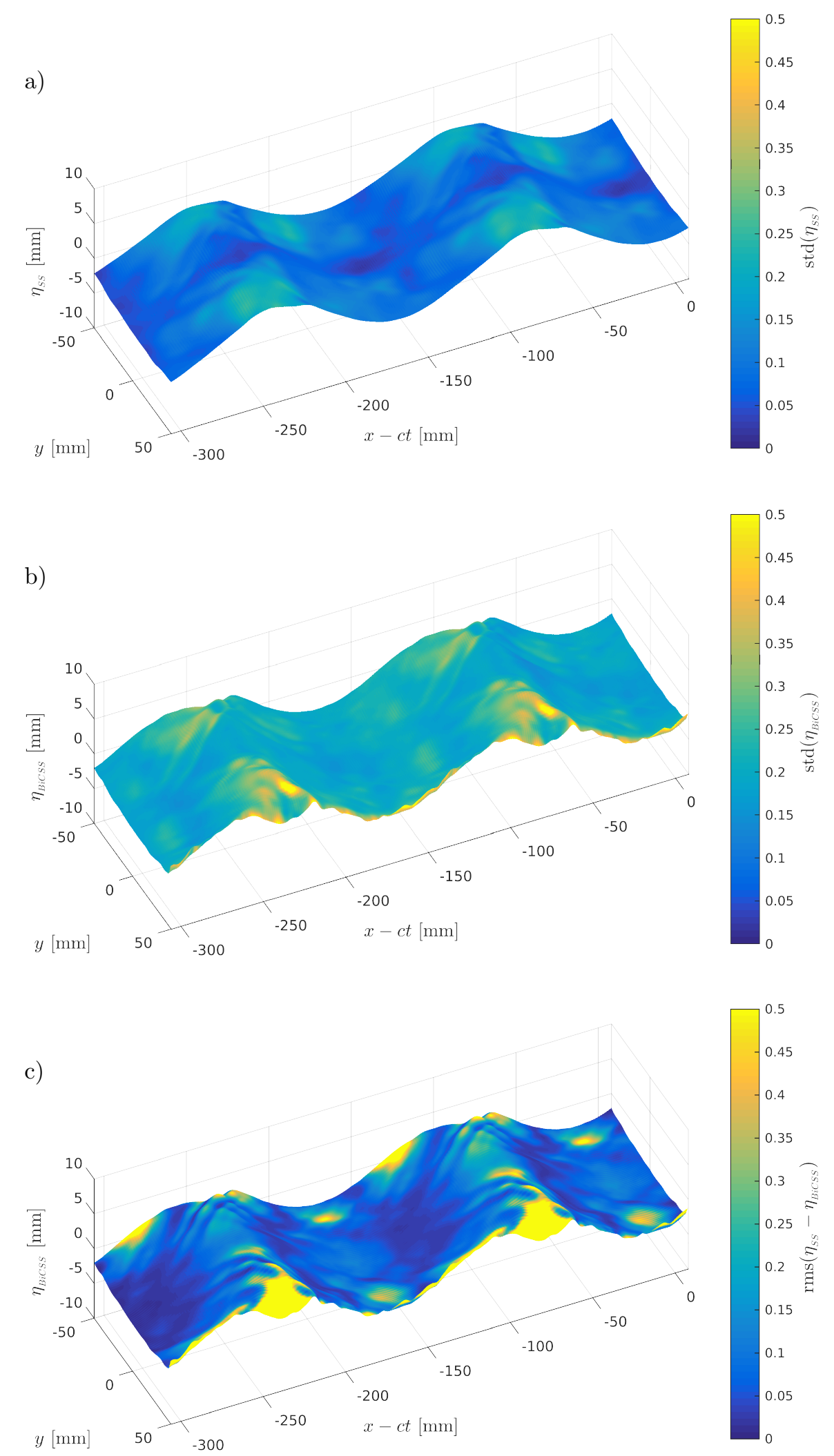

Figure 9: Phase-locked standard deviation estimation, shown by the color bar, of sinusoidal wave for a) FS-SS , b) BiCSS and c) root mean square value of difference between FS-SS and BiCSS. 
deviation

$$
\operatorname{std}(\eta)\left(x^{\prime}, y\right)=\sqrt{\left(\frac{N}{N-1}\right) \frac{\sum_{i=1}^{N} w\left(\left|x^{\prime}+c t_{i}\right| ; \varepsilon\right)\left[\eta\left(x^{\prime}+c t_{i}, y, t_{i}\right)-\bar{\eta}\left(x^{\prime}, y\right)\right]^{2}}{\sum_{i=1}^{N} w\left(\left|x^{\prime}+c t_{i}\right| ; \varepsilon\right)}}
$$

see figure 9. For FS-SS the average standard deviation of the elevation is $0.1087 \pm 0.0151 \mathrm{~mm}$, where the bounds are estimated using bootstrapping. As noted this estimate will also contain errors due to the determination of the integration constant, but possible miss some bias errors. However, the result is consistent with the error estimate found in the calibration study.

In general the determination of the integration constant would be easier and more accurate when there are multiple wavelengths within the measurement field of view. Also, the current case is much more challenging with respect to the linearization of the reconstruction problem.

It is observed, from the standard deviation in figure 9, that the highest error is near the crest. This is most likely due to light rays getting refracted into the side wall of the flume, resulting in loss of pattern to evaluate the displacement accurately.

The instantaneous fields of the two methods can be compared using the root mean square value of the difference $\overline{\operatorname{rms}\left(\eta_{S S}-\eta_{B i C S S}\right)}=0.1599 \pm 0.0580 \mathrm{~mm}$, showing that the methods are consistent. Overall the average standard deviation of the elevation using BiCSS is $0.2106 \pm 0.0369 \mathrm{~mm}$, which is larger than for FS-SS. This can be considered a trade off between resolution and accuracy.

\subsection{A vertical surface-piercing cylinder exposed to a focused wave}

A focused wave is a simple and efficient way of generating relevant laboratory measurements of wavestructure interaction. Starting with the angular center frequency $\omega_{0}$, it is possible to define the wave elevation at the focus point as

$$
\eta(t)=a_{0} e^{i \omega_{0} t} e^{-t^{2} \omega_{b}^{2}}
$$

where $a_{0}$ is the maximum amplitude, $t$ the time, and the envelope is described by the bandwidth parameter $\omega_{b}=\frac{1}{4 \sqrt{2}} \omega_{0}$. For simplicity of the calculation the complex plane is used and the actual elevation is found by taking the real part Re $(\eta)$. Furthermore, by taking the Fourier transform

$$
\hat{\eta}(\omega)=\int_{-\infty}^{\infty} \eta(0, t) e^{i \omega t} d t=\frac{a_{0} \sqrt{\pi}}{2 \omega_{b}} e^{-\frac{\left(\omega-\omega_{0}\right)^{2}}{4 \omega_{b}^{2}}}
$$

a function of the angular frequency $\omega$, it can be found that the spectrum is Gaussian. Assuming linear wave theory the spectrum can be extended in space by shifting the phase as a function of $\omega$, given by

$$
\hat{\eta}(x, \omega)=\frac{a_{0} \sqrt{\pi}}{2 \omega_{b}} e^{-\frac{\left(\omega-\omega_{0}\right)^{2}}{4 \omega_{b}^{2}}} e^{i k(\omega) x}
$$

where $x$ is the horizontal coordinate and the angular wave number $k$ is found from the linear dispersion relation $\omega^{2}=\left(g k+\sigma k^{3} / \rho\right) \tanh k h$, where $g$ is the acceleration due to gravity, $\rho$ the density of water and $\sigma$ is the surface tension. By taking the Inverse Fourier transform we get the expression for elevation for the entire wave flume

$$
\eta(x, t)=\frac{1}{2 \pi} \int_{-\infty}^{\infty} \hat{\eta}(x, \omega) e^{i \omega t} d \omega
$$

Furthermore, this can be used to calculate the paddle movement

$$
x_{a}(t)=\operatorname{Re}\left(\frac{1}{2 \pi} \int_{-\infty}^{\infty} c_{0} \hat{\eta}\left(x_{p}, \omega\right) e^{i \omega t} d \omega\right)
$$

where $x_{p}=-1.9 \mathrm{~m}$ and

$$
c_{0}=\frac{\sinh (k h) \sinh (k h)}{1 / 2(k h+\sinh (k h) \cosh (k h))},
$$



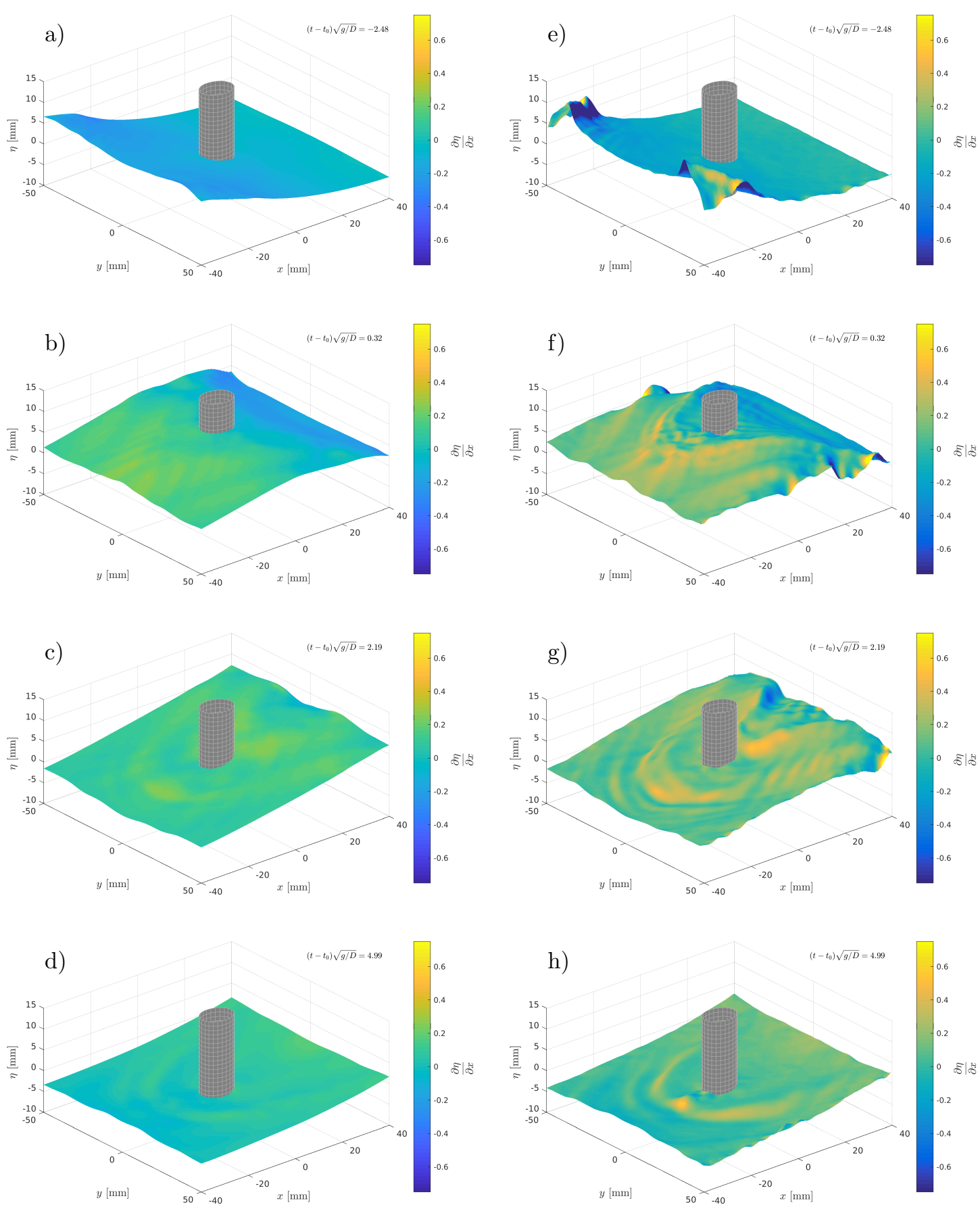

Figure 10: Reconstructed surface using FS-SS (left) and BiCSS (right) for the most steep wave, $\epsilon_{0}=0.21$ with the cylinder. Top to bottom: maximum force, maximum crest and expected timing for Type I and Type II scattered waves. 
is the 1. order transfer function (Schäffer, 1996) for a piston paddle.

The excellence of the BiCSS technique to capture small scale disturbances on waves is demonstrated by reconstructing the characteristic surface around a cylinder in waves. The cylinder was exposed to a focused wave with wave slope $\epsilon_{0}=0.2121$, where $\epsilon_{0}=a_{0} k_{0}, a_{0}$ the linear crest height, estimated from the paddle signal, and $k_{0}$ the wave number, found from the linear dispersion relation $\omega_{0}^{2}=g k_{0} \tanh k_{o} h_{w}$. For an angular center frequency $\omega_{0}=14.63$, setting the dimensionless water depth $k_{0} h_{w}=1.5$, gives a physical water depth of $h_{w}=62.5 \mathrm{~mm}$ and dimensionless wave length $k_{0} D=0.30$, where $D$ is the cylinder diameter.

Figure 10 shows the disturbed surface, reconstructed by both FS-SS (left column) and BiCSS (right column). The time $t$, with regards to $t_{0}$, where $t_{0}$ is the time when the wave force on the cylinder is zero, shortly after the passage of the wave crest, is normalized with $\sqrt{g / D}$.

First, the time for maximum load is shown in figure 10a)e) This is simultaneous with the wave zero upcrossing, where we have the maximum horizontal particle acceleration and large inertia forces. We observe a smooth surface with no disturbance, except for the extreme gradients in the two upstream corners for the BiCSS reconstructed surface (right column). These gradients are not visually observed and are considered as measurement error due to light rays being refracted outside the Synthetic Schlieren pattern or into the walls of the wave flume, with loss of information needed for accurate pattern displacement estimation.

In figure 10b)f) the time for maximum crest is shown. Here the BiCSS method reveals that the surface is strongly influenced by cross waves, where due to interaction with the main wave distinct X-shapes are created. These waves are observed both when the cylinder is present and without the cylinder in place. The frequency of these waves is equal to the first resonance frequency of the wave flume, corresponding to the wave number $\kappa=\frac{n \pi}{b}$, where $b$ is the tank width. In addition, parasitic capillary waves (LonguetHiggins, 1992; Fedorov and Melville, 1998) are observed in the front of the wave. It should be noted that due to the small scale of the experiment, both of these effects are larger than what would be expected for larger wave flumes.

In figure 10c)g), the time for minimum load is shown. This is close in time with the wave zero-down crossing, where we have a local particle acceleration maximum and large inertia forces. Here the BiCSS reconstructed surface reaveals evidence of high frequency scattered waves, referred to as Type 1 (Sheikh and Swan, 2005; Swan and Sheikh, 2015). The Type 1 scattered wavefield is driven by the run-up from the incoming wave crest approaching the cylinder face. The run-up, and subsequently wash-down, generates a concentric wave field, as primarily propagates in the upstream direction.

In figure 10d)h) the time for the following wave trough is shown. Here the BiCSS surface reveals Type II scattered waves (Sheikh and Swan, 2005; Swan and Sheikh, 2015), which appears from the motion of water around the cylinder. The motion is initiated by the wave crest, generating local disturbances on the front face, which advance around the cylinder, generating a par of non-concentric wavefronts. The overall shape of the scattered wave fields compares well with numerical simulations by Chen et al. (2014).

\section{Conclusions}

Detailed measurements of surface characteristics are an integral part of understanding complex wave phenomena and provides essential comparison data for computational modeling efforts. The present paper provides an introduction to Bichromatic Synthetic Schlieren (BiCSS) for surface measurements, a novel extension of the Free Surface Synthetic Schlieren (FS-SS) by Moisy et al. (2009).

The general procedure for these types of non-intrusive optical methods is by the use of a camera to capture images of a fixed pattern through a volume of fluid and measure the displacement of the pattern. The pattern displacement is related to surface gradients and can be used to reconstruct the surface elevation. The improved technique in BiCSS, makes use of the the difference in refraction between blue visual and near infrared (NIR) light, rather than a reference image, in obtaining the surface gradient. This improves the capture of structures on different scales, for example short waves traveling on longer waves. Furthermore, the two images, with different light wavelenghts, taken simultaneously, capture the instantaneous surface, i.e there is no time delay between images to consider.

Firstly, an experiment using phase-locked regular waves was conducted for comparing the BiCSS technique with FS-SS, analyze the properties and give an estimation of the error. To further highlight the excellence of the technique and the applicability for wave-structure interactions, a study on a vertical surface-piercing cylinder exposed to a focused wave was conducted. The obtained complex surface characteristics clearly reveals nonlinear diffraction, referred to as Type I and Type II wave scattering 
(Sheikh and Swan, 2005; Swan and Sheikh, 2015) for the steepest wave, in addition to cross waves and parasitic capillary waves.

The novel BiCSS technique demonstrated here, albeit on a relatively small scale, provides measurements on a previously unattainable level that may produce valuable data for further developments of computational models directed at, for example offshore wind farms.

\section{Appendix A Wave damper}

Due to the directional wave propagation assumptions in determination of the integration constant, it is of importance to reduce reflection from the end wall of the tank. Beaches, while excellent for deep water waves, do not perform equally well when going towards shallow water. Instead a 3D printed wave damper consisting of many of narrow $L=15 \mathrm{~cm}$ tall spikes with bases arranged using Penrose tiling was used. To evaluate the efficiency of the wave damper, the reflection index was estimated using a method by Goda and Suzuki (1977)

$$
R I=\left|\frac{\mathcal{A}}{\mathcal{B}}\right|, \quad \text { where } \mathcal{A}=\frac{\hat{\eta}(x, \omega) e^{i k_{0} \Delta x}-\hat{\eta}(x+\Delta x, \omega)}{e^{i k_{0} \Delta x}-e^{-i k_{0} \Delta x}} \text { and } \mathcal{B}=\frac{\hat{\eta}(x+\Delta x, \omega)-\hat{\eta}(x, \omega) e^{-i k_{0} \Delta x}}{e^{i k_{0} \Delta x}-e^{-i k_{0} \Delta x}} .
$$

The range of the reflection index was extended with respect to singularities by using five ultrasonic wave probes placed at $x=0 \mathrm{~cm}, 8 \mathrm{~cm}, 12 \mathrm{~cm}, 18 \mathrm{~cm}$ and $24 \mathrm{~cm}$. This creates a set with distances $\Delta x=4 \mathrm{~cm}$, $6 \mathrm{~cm}, 8 \mathrm{~cm}, 10 \mathrm{~cm}, 12 \mathrm{~cm}, 16 \mathrm{~cm}, 18 \mathrm{~cm}$ and $24 \mathrm{~cm}$ between pairs, and the refraction index is estimated as a weighted combination of all possible pair of probes, shown in figure 11. The wave damper has minimal

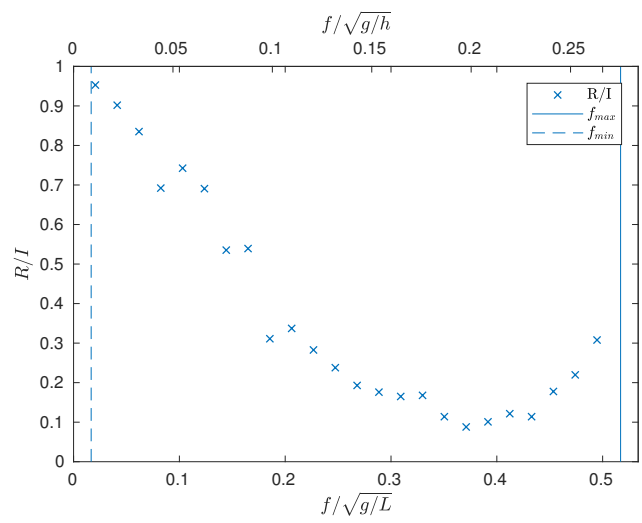

Figure 11: Reflection index for spiked wave damper. $f_{\min }$ and $f_{\max }$ indicates the singularity of the estimation method.

reflection at $\lambda \approx L$, but it should be noted that poor signal-to-noise ratio at the higher frequency range makes the estimate less accurate. In addition the estimate will also be influenced by the singularities at the end points of the range. The regular waves used in the phased-locked error estimation has a frequency $f / \sqrt{g / L}=0.3710$, at this frequency the wave damper works reasonable well with $R I \approx 0.1$. For the focus waves the center frequency $f_{0} / \sqrt{g / L}=0.2879$, in this case the reflection is of less importance since the measurement is taken before the reflection reaches the measurement section.

\section{Acknowledgements}

The study has been carried out with financial support from Stiftelsen Det Norske Veritas, DNV GL and The Research Council of Norway through grant number 231491: Developments in Optical Measurement Technologies (DOMT). Technical assistance during the experimental work by Head Engineer Olav Gundersen is gratefully acknowledged.

\section{References}

Aureli, F., Dazzi, S., Maranzoni, A., Mignosa, P., 2014. A combined colour-infrared imaging technique for measuring water surface over non-horizontal bottom. Experiments in fluids 55, 1-14.

Benetazzo, A., Barbariol, F., Bergamasco, F., Torsello, A., Carniel, S., Sclavo, M., 2016. Stereo wave imaging from moving vessels: Practical use and applications. Coastal Engineering 109, 114-127. 
Chaplin, J.R., Rainey, R.C.T., Yemm, R.W., 1997. Ringing of a vertical cylinder in waves. Journal of Fluid Mechanics 350, 119-147.

Chen, L.F., Zang, J., Hillis, A.J., Morgan, G.C.J., Plummer, A.R., 2014. Numerical investigation of wave--structure interaction using openfoam. Ocean Engineering 88, 91-109.

Ciddor, P.E., 1996. Refractive index of air: new equations for the visible and near infrared. Applied optics 35, $1566-1573$.

Daimon, M., Masumura, A., 2007. Measurement of the refractive index of distilled water from the near-infrared region to the ultraviolet region. Applied optics 46, 3811-3820.

Dalziel, S., Hughes, G.O., Sutherland, B.R., 2000. Whole-field density measurements by 'synthetic schlieren'. Experiments in Fluids 28, 322-335.

D'Errico, J., 2006. Inverse (integrated gradient). Matlab Central.

Fedorov, A.V., Melville, W.K., 1998. Nonlinear gravity-capillary waves with forcing and dissipation. Journal of Fluid Mechanics 354, 1-42.

Goda, Y., Suzuki, Y., 1977. Estimation of incident and reflected waves in random wave experiments, in: Coastal Engineering 1976, pp. 828-845.

Gomit, G., Chatellier, L., Calluaud, D., David, L., 2013. Free surface measurement by stereo-refraction. Experiments in fluids $54,1-11$.

Jähne, B., 1990. Two-dimensional wave nulllber spectra of small-scale water surface waves. Journal of Geophysical Research $95,11-531$.

Jähne, B., Schmidt, M., Rocholz, R., 2005. Combined optical slope/height measurements of short wind waves: principle and calibration. Measurement Science and Technology 16, 1937.

Kiefhaber, D., Reith, S., Rocholz, R., Jähne, B., 2014. High-speed imaging of short wind waves by shape from refraction. Journal of the European Optical Society-Rapid publications 9.

Kolaas, J., 2016. Getting started with HydrolabPIV v1.0. Preprint series. Research Report in Mechanics

Kolaas, J., Smith, L., Sveen, J.K., Jensen, A., 2016. Bichromatic synthetic schlieren for surface measurement, in: Proceedings of the 18th International Symposium on the Application of Laser and Imaging Techniques to Fluid Mechanics.

Kristiansen, T., Faltinsen, O.M., 2017. Higher harmonic wave loads on a vertical cylinder in finite water depth. Journal of Fluid Mechanics 833, 773-805.

Longuet-Higgins, M.S., 1992. Capillary rollers and bores. Journal of Fluid Mechanics 240, 659-679.

Meier, G., 2002. Computerized background-oriented schlieren. Experiments in Fluids 33, 181-187.

Moisy, F., Rabaud, M., Salsac, K., 2009. A synthetic schlieren method for the measurement of the topography of a liquid interface. Experiments in Fluids 46, 1021-1036.

Paquier, A., Moisy, F., Rabaud, M., 2015. Surface deformations and wave generation by wind blowing over a viscous liquid. Physics of Fluids 27, 122103.

Paulsen, B.T., Bredmose, H., Bingham, H.B., 2014a. An efficient domain decomposition strategy for wave loads on surface piercing circular cylinders. Coastal Engineering 86, 57-76.

Paulsen, B.T., Bredmose, H., Bingham, H.B., Jacobsen, N.G., 2014b. Forcing of a bottom-mounted circular cylinder by steep regular water waves at finite depth. Journal of Fluid Mechanics 755, 1-34.

Rainey, R.C.T., 2007. Weak or strong nonlinearity: the vital issue. Journal of Engineering Mathematics 58, 229-249.

Schäffer, H.A., 1996. Second-order wavemaker theory for irregular waves. Ocean Engineering 23, 47-88.

Sheikh, R., Swan, C., 2005. The interaction between steep waves and a vertical, surface-piercing column. J. Offshore Mech. Arct. 127, 31-38.

Sultanova, N., Kasarova, S., Nikolov, I., 2009. Dispersion proper ties of optical polymers. Acta Physica Polonica-Series A General Physics 19, 585.

Sutherland, B.R., Dalziel, S.B., Hughes, G.O., Linden, P., 1999. Visualization and measurement of internal waves by 'synthetic schlieren'. part 1. vertically oscillating cylinder. Journal of fluid mechanics 390, 93-126.

Swan, C., Sheikh, R., 2015. The interaction between steep waves and a surface-piercing column. Phil. Trans. R. Soc. A $373,20140114$.

Toffoli, A., Gramstad, O., Trulsen, K., Monbaliu, J., Bitner-Gregersen, E., Onorato, M., 2010. Evolution of weakly nonlinear random directional waves: laboratory experiments and numerical simulations. Journal of Fluid Mechanics $664,313-336$.

Zappa, C.J., Banner, M.L., Schultz, H., Corrada-Emmanuel, A., Wolff, L.B., Yalcin, J., 2008. Retrieval of short ocean wave slope using polarimetric imaging. Measurement Science and Technology 19, 055503. 